\title{
Type II cytokines impair host defense against an intracellular fungal pathogen by amplifying macrophage generation of IL-33
}

\author{
A Verma ${ }^{1,2}$, DN Kroetz ${ }^{3}, \mathrm{JL}_{\text {Tweedle }}$ T,4 $^{2}$ and GS Deepe $\mathrm{Jr}^{2,5}$
}

Interleukin (IL)-4 subverts protective immunity to multiple intracellular pathogens, including the fungus Histoplasma capsulatum. Previously, we reported that $H$. capsulatum-challenged CCR2 ${ }^{-1-}$ mice manifest elevated pulmonary fungal burden owing to exaggerated IL-4. Paradoxical to our anticipation in IL-33 driving IL-4, we discovered that the latter prompted IL-33 in mutant mice. In infected CCR2 ${ }^{-1-}$ animals, amplified IL-33 succeeded the heightened IL-4 response and inhibition of IL-4 signaling decreased IL-33. Moreover, macrophages, but not epithelial cells or dendritic cells, from these mice expressed higher IL-33 in comparison with controls. Dissection of mechanisms that promulgated IL-33 revealed type-Il cytokines and $H$. capsulatum synergistically elicited an IL-33 response in macrophages via signal transducer and activator of transcription factor 6/interferon-regulatory factor-4 and Dectin-1 pathways, respectively. Neutralizing IL-33 in CCR2 ${ }^{-/-}$animals, but not controls, enhanced their resistance to histoplasmosis. Thus we describe a previously unrecognized role for IL-4 in instigating IL-33 in macrophages. Furthermore, in the presence of intracellular fungal pathogens, the type-II cytokine-driven IL-33 response impairs immunity.

\section{INTRODUCTION}

The prototypical T helper type 2 (Th2) cytokine, interleukin (IL)-4, is vital for host immunity against helminth infections ${ }^{1}$ and for wound healing and tissue repair. ${ }^{2}$ Conversely, the cytokine has deleterious effects in the setting of intracellular infections $\mathrm{s}^{3,4}$ and allergic disorders, such as asthma and atopic dermatitis. ${ }^{5,6}$ IL-4 polarizes macrophages to an alternatively activated phenotype, thereby providing a congenial environment for intracellular pathogens to proliferate freely. ${ }^{7}$ Another key attribute of IL-4 is to differentiate naive $\mathrm{CD} 4{ }^{+} \mathrm{T}$ cells to the Th2 lineage; however, undesired activation of Th2 cells during certain microbial infections suppresses Th1 immune responses. ${ }^{8}$ Recently, considerable interest has been generated in the epithelial derived cytokines IL-25, IL-33, and thymic stromal lymphopoietin that are important in initiating IL-4. ${ }^{9}$ IL-33, in particular, acts on a variety of innate and adaptive immune cell populations, including basophils, dendritic cells, macrophages, and $\mathrm{CD}^{+} \mathrm{T}$ cells, via its transmembrane receptor ST2 to induce Th2 immune responses. ${ }^{10}$

Histoplasma capsulatum is a dimorphic intracellular fungal pathogen and the etiologic agent of histoplasmosis. It is found worldwide, but there are regions of notably high incidence of infections, such as the Ohio and Mississippi River Valleys in the USA and regions of South America. ${ }^{11}$ Each year, in the United States alone, it is estimated that up to 25,000 people develop lifethreatening infections in endemic regions and 10 -fold more individuals acquire mild or asymptomatic disease. ${ }^{12}$ To establish extensive infection in humans, H. capsulatum transforms into its unicellular yeast phase and disseminates to other organs using macrophages as vehicles. Acute fungal infection in immunocompetent hosts is usually efficiently resolved; however, the more severe symptomatic infections occur in the setting of immunosuppression, including patients with AIDS and individuals chronically receiving immunosuppressive drugs. ${ }^{13}$

${ }^{1}$ Division of Immunobiology, Cincinnati Children's Hospital Medical Center, University of Cincinnati, Cincinnati, Ohio, USA. ${ }^{2}$ Division of Infectious Diseases, College of Medicine, University of Cincinnati, Cincinnati, Ohio, USA. ${ }^{3}$ Department of Pathology, University of Michigan, Ann Arbor, Michigan, USA. ${ }^{4}$ Department of Pathobiology and Molecular Medicine, University of Cincinnati, Cincinnati, Ohio, USA and ${ }^{5}$ Veterans Affairs Hospital, Cincinnati, Ohio, USA. Correspondence: GS Deepe (george.deepe@uc.edu)

Received 18 April 2014; accepted 15 July 2014; published online 13 August 2014. doi:10.1038/mi.2014.75 
The successful clearance of $H$. capsulatum infection is largely based on the coordinated action of innate and adaptive arm of the immune system. The pathogen initially triggers a host response by activating innate immunity through patternrecognition receptors such as CR3 and Dectin-1 expressed on phagocytes. ${ }^{14,15}$ This is followed by a robust Th1 immune response characterized by proinflammatory cytokines interferon- $\gamma$, tumor necrosis factor (TNF)- $\alpha$, and granulocyte macrophage colony-stimulating factor (GM-CSF) and classical activation of macrophages. ${ }^{16}$ In contrast, a defective immune response or an overproduction of Th2 cytokines leads to alternative macrophage activation and ultimately increased susceptibility to $H$. capsulatum. ${ }^{3}$

In the previous work, we reported that in the absence of chemokine receptor 2 (CCR2), mice exhibit enhanced susceptibility to $H$. capsulatum in comparison with wild-type (WT) controls. ${ }^{3}$ The major defect in immunity in these mutant mice is the heightened production of IL- 4 in the lungs that starts as early as day 3 of infection. In this manuscript, we report that CCR2 ${ }^{-1-}$ mice mounted an elevated IL-33 response after initiation of the IL-4 response. Paradoxical to the function of IL-33 in initiating a Th2 immune response, we discovered that IL-4 induced it in the lungs of infected CCR2 ${ }^{-1-}$ mice. Moreover, macrophages were the central source of IL-33 in these mice. Dissection of the molecular mechanism revealed that IL-4 and H. capsulatum synergistically evoked IL-33 in macrophages and the signaling pathway was dependent on signal transducer and activator of transcription factor 6 (STAT6)/interferon-regulatory factor-4 (IRF-4) and Dectin1, respectively. Finally, IL-33 exhibited a deleterious role in $H$. capsulatum infection by inducing alternatively activated phenotype in macrophages, and neutralizing its effects in the
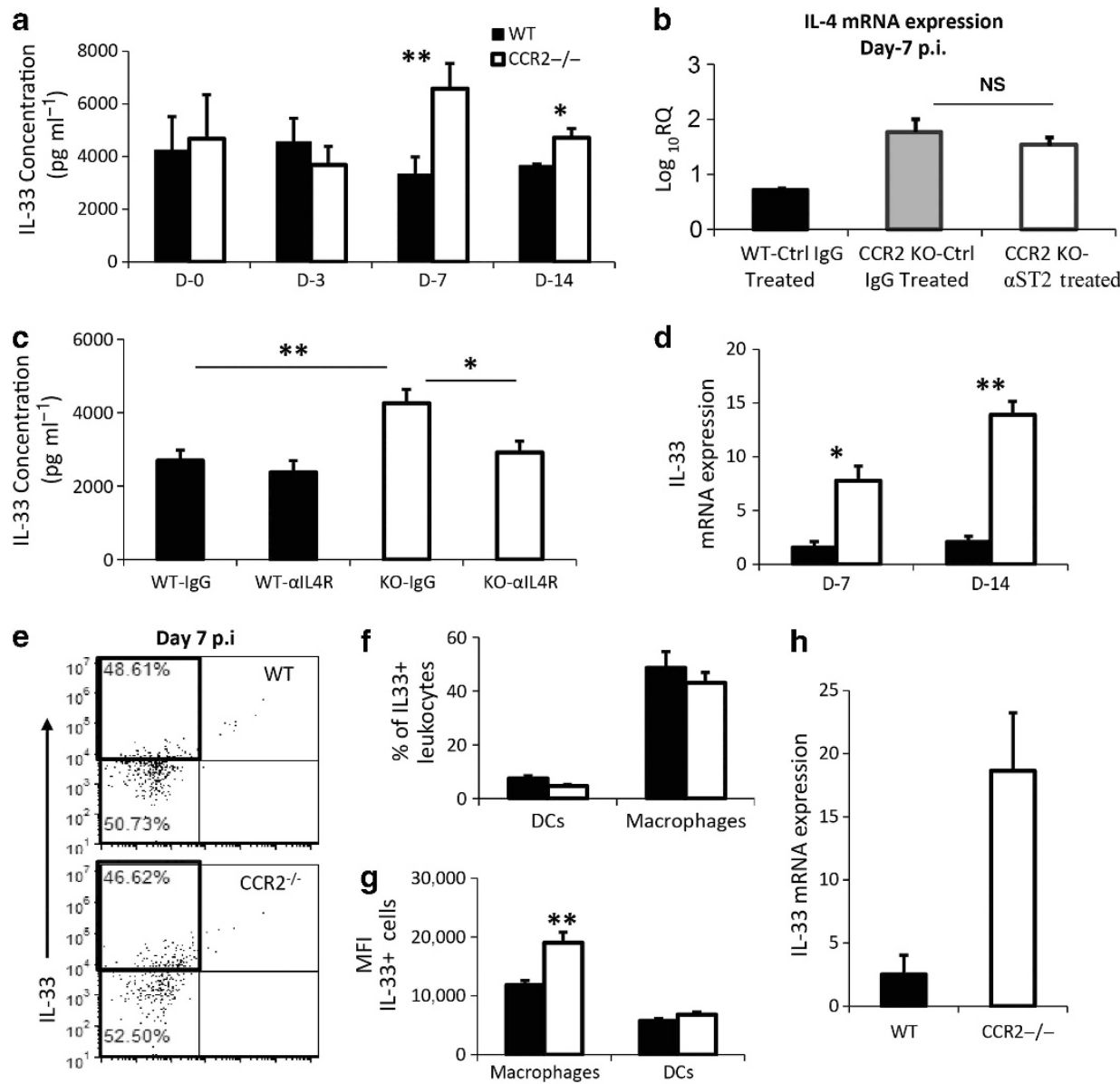

h

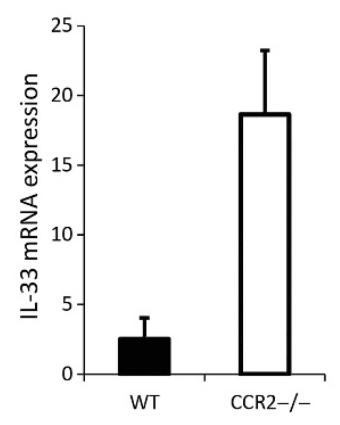

Figure 1 Interleukin (IL)-4 prompts an IL-33 response in macrophages in vivo. (a) IL-33 protein concentration measured by enzyme-linked immunosorbent assay in lung homogenates of WT and CCR2 ${ }^{-1-}$ mice at the indicated time points. (b) IL-4 mRNA expression in whole lung homogenates of mice treated with anti-ST2 or isotype control antibody. Transcription is expressed as $\log _{10}$ relative quantification normalized to uninfected wild-type (WT) lung. (c) IL-33 protein concentration measured at day-7 postinfection (p.i.) in lung homogenates of mice treated with anti-IL-4R $\alpha$ or isotype control antibody. (d) IL-33 mRNA expression in isolated lung leukocytes from WT and CCR2 ${ }^{-1}$ mice at the indicated time points. Data are normalized to lung leukocytes from uninfected WT animals. (e) Intracellular staining of IL-33 in pulmonary macrophages from WT and CCR2 ${ }^{-1-}$ mice at 7 days p.i. (gating strategy depicted in Supplementary Figure S1B); $x$ axis depicts empty channel. Representative plot of one of eight mice from two independent experiments. ( $\mathbf{f}$ and $\mathbf{g}$ ) Analysis of IL-33 expression and mean fluorescence intensity (MFI) by flow cytometry in macrophages and dendritic cells (DCs) at day-7 p.i. (h) IL-33 mRNA expression in sorted F4/80 ${ }^{+}$macrophages from lungs of WT and CCR2 ${ }^{-1-}$ mice at 7 days p.i. Data are normalized to uninfected WT macrophages. Pooled values of 8-12 mice from one of two similar experiments. For all other in vivo experiments, data are represented as mean \pm s.e.m., $n=6-8$ from two independent experiments. NS, not significant. 
CCR2 $2^{-1-}$ mice resulted in decreased fungal burden in the lungs. Thus we report that IL-4 and $H$. capsulatum are inducers of IL-33 in macrophages, and the IL-4 driven IL-33 response during fungal infection orchestrates detrimental effects in the host that, in turn, leads to a collapse in immunity to the disease.

\section{RESULTS}

\section{Induction of IL-33 by IL-4 in vivo}

H. capsulatum-infected CCR2 $2^{-1-}$ mice display exaggerated levels of IL-4 in the lungs beginning as early as day $3 .^{3}$ Using these mutant mice as a model for dysregulated IL-4 in an intracellular infection, we explored the cytokines associated with regulation of IL-4. Thymic stromal lymphopoietin, IL-25 and IL-33 have been implicated in initiating a type II immune response. ${ }^{17}$ To investigate their contribution in evoking the dominant IL-4 response in CCR2 ${ }^{-1-}$ mice, we measured concentrations in lung homogenates between days 0 and 3 of infection. This particular time frame was chosen because CCR $2^{-1-}$ mice exhibit elevated IL- 4 beginning as early as day 3 of $H$. capsulatum infection. No differences were observed in protein concentrations of IL-25 $\left(<50 \mathrm{pg} \mathrm{ml}^{-1}\right)$ and thymic stromal lymphopoietin $\left(<25 \mathrm{pg} \mathrm{ml}^{-1}\right)$ between days $0-3$ in WT and CCR2 $2^{-1-}$ mice. Thus, it was unlikely that these cytokines instigated the exaggerated IL-4 response. We did observe slightly upregulated IL-33 transcripts in lungs of CCR2 $2^{-1-}$ mice at day 3 of infection $(3.2 \pm 0.34$ fold vs. $1.9 \pm 0.18$ fold in WT mice; $P<0.05$ ), but protein concentrations were similar between the two groups (Figure 1a).
To examine if IL-33 triggered IL-4 in CCR2 ${ }^{-1-}$ mice, we administered ST2-blocking antibody. Similar pulmonary IL-4 protein concentration following anti-ST2 or control IgG antibody treatment led us to conclude that IL-33 did not evoke the amplified IL-4 response in mutant mice (Figure 1b). Serendipitously, we discovered augmented IL-33 protein in the lungs of CCR2 ${ }^{-1-}$ mice at days 7 and 14 post-infection that succeeded, rather than preceded the exaggerated IL-4 response (Figure 1a). The unexpected kinetics of IL- 4 and IL-33 prompted us to determine if IL-4 elicited an IL-33 response. We treated CCR2 ${ }^{-1-}$ mice with IL-4R $\alpha$-blocking antibody prior to infection and analyzed pulmonary IL-33 concentration 7 days later. Although using anti-IL- $4 \mathrm{R} \alpha$ antibody disrupts IL-4 and IL-13 signaling, IL-13 is not upregulated in H. capsulatum-infected CCR2 ${ }^{-1-}$ mice. ${ }^{3}$ Inhibition of IL-4 engagement with its receptor decreased IL-33 in the lungs (Figure 1c).

IL-33 is generated by lung epithelial cells in response to helminth and fungal infections. ${ }^{18,19}$ To investigate the cellular source of this cytokine in the lungs, its expression in pulmonary epithelial cells was analyzed by flow cytometry. WT and CCR2 ${ }^{-1-}$ mice displayed similar percentages and mean fluorescence intensity of $\mathrm{IL}-33^{+}$epithelial cells (CD45 ${ }^{-}$ $\mathrm{MHCII}^{-} \mathrm{EpCAM}^{+}$) at 7 days of infection (Supplementary Figure S1A online). Although pulmonary epithelial cells are a source of IL-33, leukocytes synthesize it too. IL-33 was upregulated in leukocytes at 7 and 14 days after infection in CCR2 ${ }^{-1-}$ mice (Figure 1d). Among leukocytes, macrophages
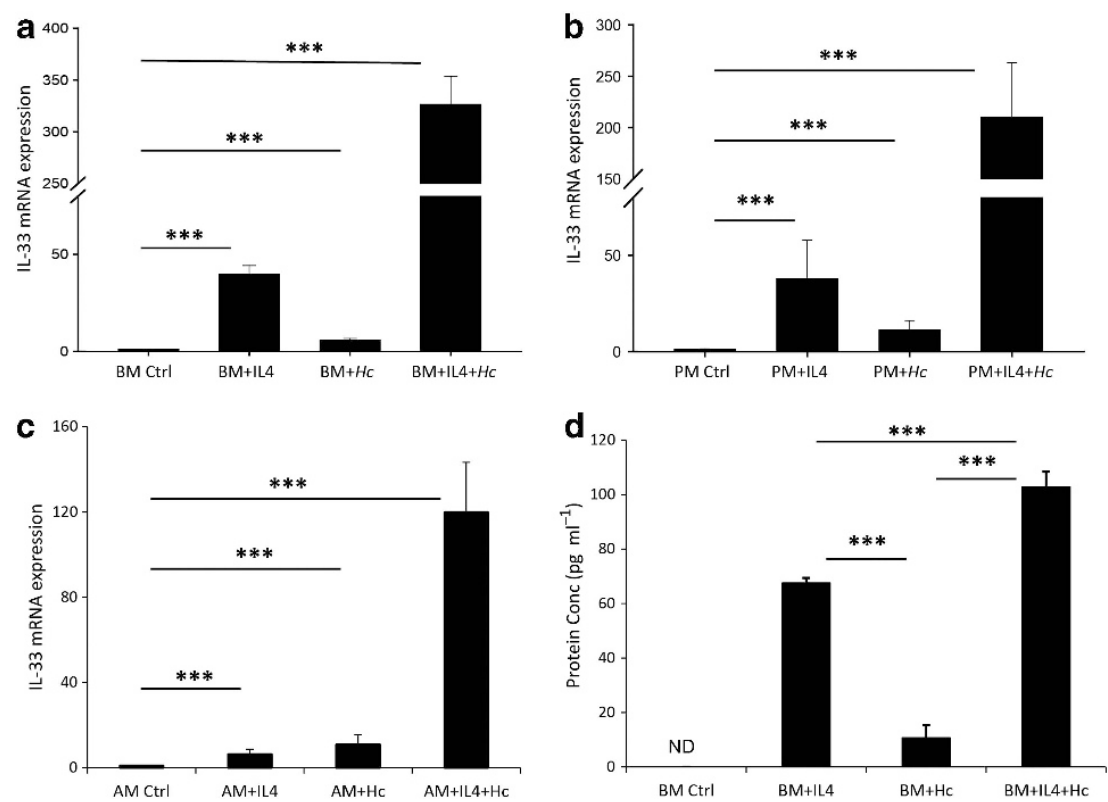

Figure 2 Synergistic induction of interleukin (IL)-33 by IL-4 and $H$. capsulatum $(H c)$ in vitro. (a) IL-33 mRNA expression measured by quantitative realtime reverse transcription PCR in wild-type (WT) bone marrow-derived macrophages (BM) that were exposed to IL-4 (10 $\mathrm{ng} \mathrm{ml}^{-1}$ ) or $\mathrm{H}_{\text {. capsulatum }(5}$ multiplicity of infection (MOI)) or both for $24 \mathrm{~h}$. All macrophages exposed to IL-4 or IL-4 $+\mathrm{H}$. capsulatum were initially primed with IL-4 (10 $\mathrm{ng} \mathrm{ml}^{-1}$ ) for $24 \mathrm{~h}$. Values are normalized to untreated macrophages. All the treatment groups are significantly different $(P<0.05)$ from each other. (b and $\mathbf{c})$ IL-33 mRNA expression in resident peritoneal macrophages (PM) and alveolar macrophages (AM) isolated from WT mice that were exposed to IL-4 $\left(10 \mathrm{ng} \mathrm{ml}^{-1}\right.$ ) or $\mathrm{H}$. capsulatum $(5 \mathrm{MOI})$ or both in vitro for $24 \mathrm{~h}$. All the treatment groups are significantly different $(P<0.05)$ from each other. (d) Intracellular IL-33 protein content in whole-cell lysates (cells lysed with deionized water) measured by enzyme-linked immunosorbent assay after $24 \mathrm{~h}$ of exposure to the stimulus. All the treatment groups are significantly different $(P<0.05)$ from each other. Values depicted in all the above experiments are mean \pm s.e.m. from at least five independent experiments. 
and dendritic cells are associated with IL-33 production in lungs. ${ }^{19,20}$ Intracellular staining revealed that WT and CCR2 ${ }^{-1-}$ mice displayed similar percentages of $\mathrm{IL}-33^{+}$ macrophages, but the latter expressed a higher IL-33 mean fluorescence intensity (Supplementary Figure S1B and Figure 1e-g). To corroborate this finding, we sorted $\mathrm{F} 4 / 80^{+}$macrophages from the lungs of infected WT and mutant mice and measured IL-33 expression. Macrophages isolated from CCR2 ${ }^{-1-}$ mice exhibited increased IL-33 mRNA (Figure 1h). No differences in the total percentage and mean fluorescence intensity of IL- $33^{+}$dendritic cells were observed (Figure 1f,g). These findings strongly suggested that IL-4 induces IL-33 in macrophages during $H$. capsulatum infection in vivo.

\section{IL-4 and $\boldsymbol{H}$. capsulatum instigate a strong synergistic IL-33 response in macrophages in vitro}

To dissect the signaling pathway of how IL-4 drives IL-33, an in vitro system was established to study IL-33 expression in macrophages following IL-4 exposure. Stimulation of bone marrow-derived macrophages with IL-4 or infection with $H$. capsulatum yeasts resulted in a modest induction of IL-33 mRNA. IL-4-treated infected macrophages manifested a synergistic IL-33 mRNA response (Figure 2a). Similarly, peritoneal macrophages and alveolar macrophages synthesized IL-33 when exposed to IL-4 or $H$. capsulatum or both (Figure 2b,c). CCR2 ${ }^{-1-}$ macrophages generated an IL-33 response comparable with WT cells following incubation with IL-4 or H. capsulatum or both (Supplementary Figure S2A). IL-33 in the lungs of WT and CCR2 $2^{-/-}$mice was similar prior to infection (Figure 1b). These findings demonstrated that the exaggerated IL-33 response in CCR2 ${ }^{-1}$ animals following infection was a result of heightened production of IL-4 rather than an intrinsic defect caused by the lack of CCR2. Thus we utilized WT macrophages for the subsequent experiments. To examine whether this response was restricted to $H$. capsulatum, macrophages were infected with a phylogenetically related fungus, Blastomyces dermatitidis. An analogous IL-33 expression profile was noted when macrophages were infected with $B$. dermatitidis alone or with IL-4 (Supplementary Figure S2B).

Similar to other members in the IL-1 family, IL-33 lacks a classical secretory leader sequence that prevents its active release from the cells. ${ }^{10}$ We did not detect IL-33 in culture supernatants. To ascertain whether the protein was produced, we lysed cells with water and assessed the IL-33 concentrations. The intracellular protein concentration mirrored the mRNA response when macrophages were incubated with IL-4 or $H$. capsulatum or both (Figure $2 \mathbf{d}$ ). There was no secretory defect in these infected macrophages as IL-1 $\beta$ and TNF- $\alpha$ were detected in culture supernatants. These cytokines were readily detected $24 \mathrm{~h}$ after infection (IL-1 $\beta, 75 \pm 4 \mathrm{pg} \mathrm{ml}^{-1}$; and TNF$\left.\alpha, 174 \pm 44 \mathrm{pg} \mathrm{ml}^{-1} ; n=5\right)$.

We investigated the kinetics of IL-33 transcription by IL-4. Macrophages required treatment with IL-4 for at least $48 \mathrm{~h}$ to produce a maximal IL-33 response (Supplementary
Figure S2C). The magnitude of the response directly correlated with the quantity of IL-4 added to the cultures (Supplementary Figure S2D). Infecting macrophages with an increasing multiplicity of infection (MOI) of $H$. capsulatum yeasts augmented IL-33 mRNA (Supplementary Figure S2E). Thus IL-4 or $H$. capsulatum alone elicits modest upregulation of IL-33 in macrophages; however, when both are present, macrophages mount a strong synergistic IL-33 response.

\section{IL-4-induced IL-33 response in macrophages is dependent on STAT6 and IRF-4}

IL-4 mediates its downstream effects through the transcription factor STAT6. ${ }^{21}$ To determine whether this factor was necessary for elicitation of IL-33, STAT6 ${ }^{-1-}$ macrophages were subjected to IL-4 or IL-4 plus $H$. capsulatum. The IL-33 response was abolished in these macrophages when stimulated with IL-4 (Figure 3a). The synergistic IL-33 response in STAT6 $^{-1-}$ cells was lost when they were exposed to a combination of IL-4 and H. capsulatum. Treatment of cells with
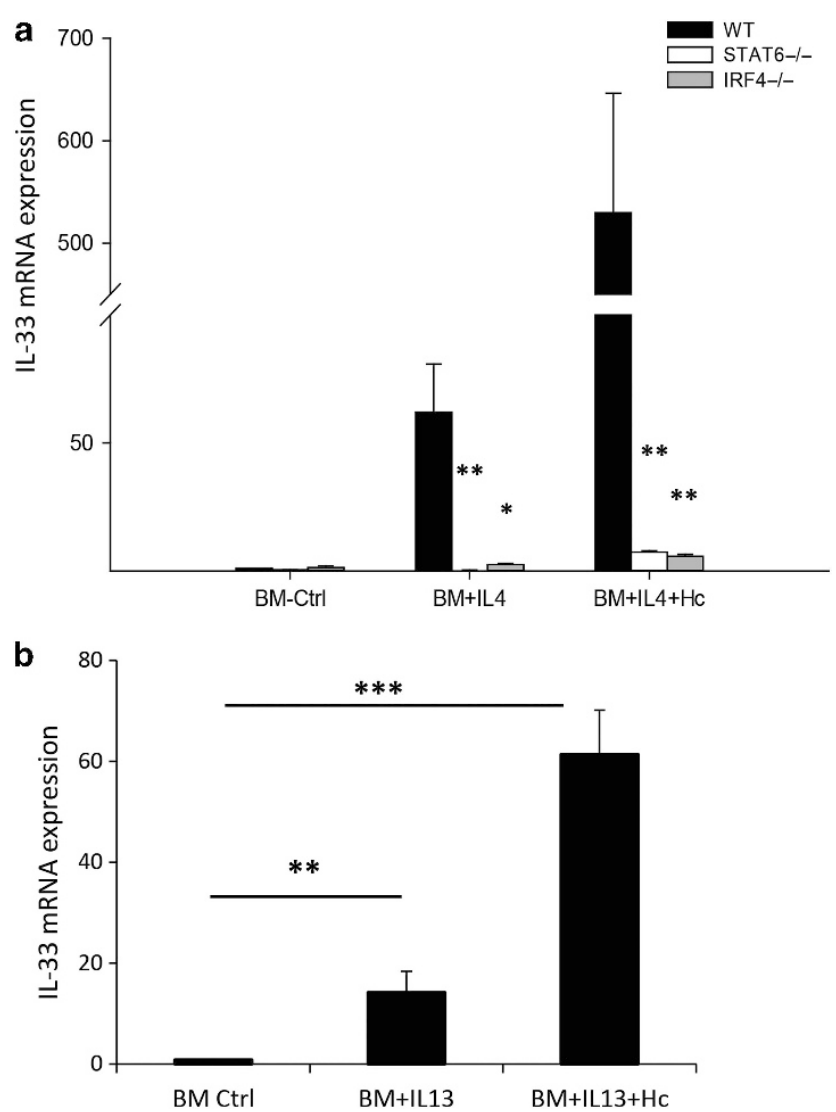

Figure 3 Interleukin (IL)-4-induced IL-33 response is dependent on signal transducer and activator of transcription factor 6 (STAT6) and interferon-regulatory factor-4 (IRF-4). (a) IL-33 mRNA expression in bone marrow-derived macrophages (BM) from wild-type (WT), STAT6 ${ }^{-1-}$, and IRF-4 $4^{-1}$ animals that were subjected to IL-4 treatment or $H$. capsulatum infection or both in vitro for $24 \mathrm{~h}$. Values are normalized to untreated WT macrophages. Data are mean \pm s.e.m. from at least three independent experiments. (b) IL-33 transcription measured in WT macrophages exposed to IL-13 $\left(10 \mathrm{ng} \mathrm{ml}^{-1}\right)$ or IL-13 plus $H$. capsulatum ( 5 multiplicity of infection) for $24 \mathrm{~h}$. Data are mean \pm s.e.m. from five independent experiments. 
IL-13, another cytokine that activates STAT6, produced effects analogous to IL-4 (Figure $3 \mathbf{b}$ ).

IRF-4 is a STAT6-inducible transcription factor that is rapidly transcribed when macrophages are stimulated with IL-4. ${ }^{22}$ To determine whether IRF-4 was requisite for the enhanced IL-33 response, IRF-4 ${ }^{-/-}$macrophages were exposed to IL-4 or $H$. capsulatum or both. IL-33 expression in these cells was comparable with STAT6 ${ }^{-/}$macrophages (Figure 3a). Hence, the induction of IL-33 is dependent on an IL-4/STAT6/IRF-4 signaling cascade in macrophages.

\section{Synergistic induction of IL-33 is not a result of increased expression of IL-4R $\alpha$ or autocrine IL-4 and IL-13 generated by macrophages during infection}

The intracellular pathogen Mycobacterium tuberculosis induces the expression of IL- $4 \mathrm{R} \alpha$ in macrophages. ${ }^{23}$ A possible explanation for the synergistic elicitation of IL-33 was that $H$. capsulatum upregulated the expression of IL- $4 \mathrm{R} \alpha$ on the macrophage surface, which, in turn, potentiated reactivity to IL-4. To test this, expression of IL- $4 \mathrm{R} \alpha$ was analyzed in macrophages infected with $H$. capsulatum. Exposure to the fungus did not significantly upregulate IL- $4 \mathrm{R} \alpha$ mRNA compared with controls (Figure 4a). Another possibility was that infected macrophages secreted IL-4 and/or IL-13 that acted in an autocrine manner to drive IL-33. We assessed IL-4 and IL-13 in culture supernatants of infected macrophages but did not detect either (IL-4 and IL-13<2 $\mathrm{pg} \mathrm{ml}^{-1}$ ). Western blotting analysis of infected cell lysates indicated that STAT6 was not phosphorylated during the course of infection (Figure $4 \mathbf{b}$ ). We exposed IL-4 ${ }^{-1}$ macrophages to IL-4 or H. capsulatum or both and observed an analogous IL-33 expression profile as WT cells (Figure 4c). To demonstrate that the synergistic evocation of IL-33 was not a result of autocrine IL- 4 or IL-13, IL-4R $\alpha^{-1-}$ macrophages were utilized. These did not mount an IL-33 response when stimulated with IL- 4 alone but had a similar induction of IL-33 as the WT macrophages upon infection with H. capsulatum (Figure 4d). The synergistic elicitation of IL-33 was not a result of increased expression of IL- $4 \mathrm{R} \alpha$ or autocrine IL-4/IL-13 produced by infected macrophages.

\section{$H$. capsulatum-induced IL-33 response in macrophages is dependent on Dectin-1 signaling pathway}

Dectin-1 exerts an auxiliary role in triggering innate responses when macrophages interact with $H$. capsulatum yeasts in vitro. ${ }^{15}$ The significance of Dectin-1 signaling pathway in the inception of IL-33 response was examined following $H$. capsulatum exposure. We infected Dectin-1 ${ }^{-1-}$ macrophages treated with IL-4 or vehicle for $24 \mathrm{~h}$ and analyzed the expression of IL-33. The synergistic IL-33 response in Dectin$1^{-/-}$macrophages was greatly diminished compared with WT cells (Figure 5a). A potential caveat with this experiment was an attenuation in binding of $H$. capsulatum yeasts to Dectin-1 ${ }^{-1-}$ macrophages and hence a subsequent decrease in phagocytosis. We quantified the association index of green fluorescent a
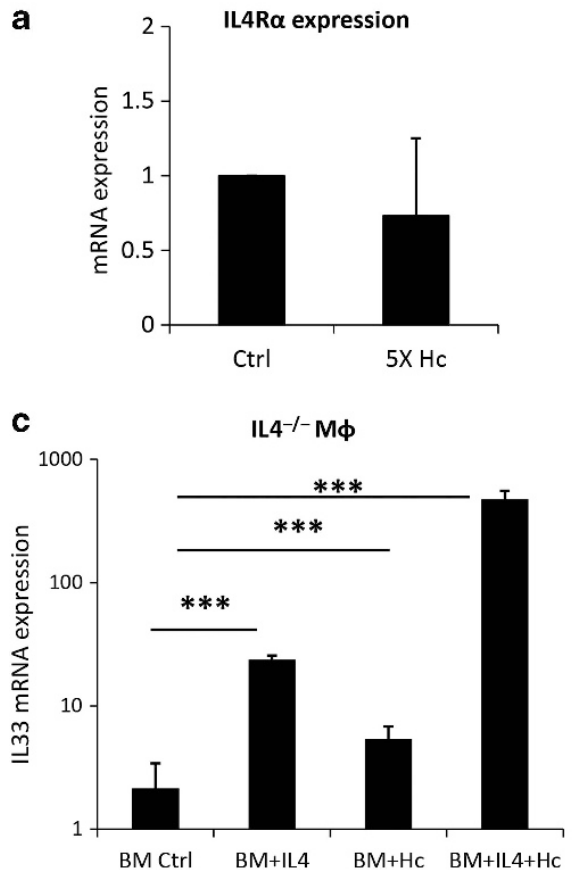

b

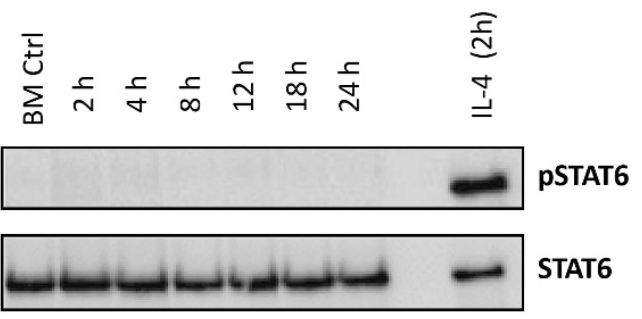

d

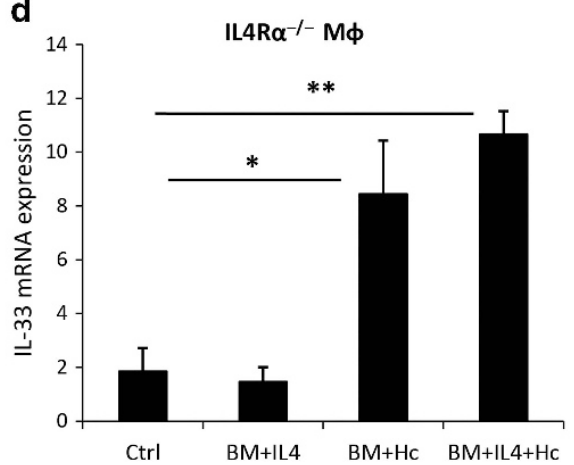

Figure 4 Synergistic induction of interleukin (IL)-33 is not a result of increased expression of IL-4R $\alpha$ or autocrine IL-4/IL-13. (a) IL-4R $\alpha$ mRNA expression in macrophages quantified by quantitative real-time reverse transcription PCR after $24 \mathrm{~h}$ of $H$. capsulatum infection ( 5 multiplicity of infection). Data are mean \pm s.e.m. from three independent experiments. (b) Immunoblot of phospho-signal transducer and activator of transcription factor 6 (phospho-STAT6) and total STAT6 from whole-cell lysates of macrophages infected with H. capsulatum for the indicated time. Macrophages stimulated with IL-4 were used as a positive control. Representative blot of three similar experiments. (c and d) IL-33 mRNA expression in IL-4 ${ }^{-1-}$ and IL-4R $\alpha^{-1-}$ macrophages exposed to IL-4 or H. capsulatum or both for $24 \mathrm{~h}$. Values are normalized to unstimulated wild-type macrophages. Data are mean \pm s.e.m. from three independent experiments. All the treatment groups of $\mathrm{IL}-4^{-1}$ macrophages are significantly different $(P<0.05)$ from each other. 

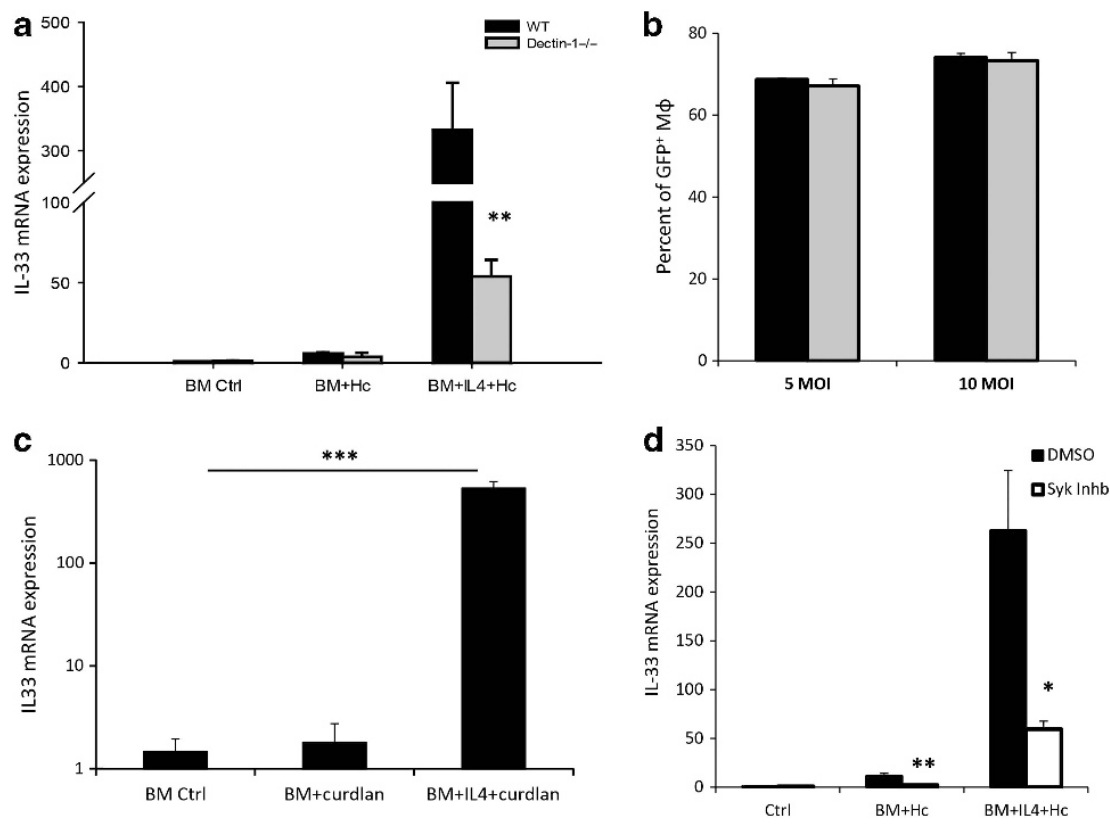

Figure $5 H$. capsulatum-induced interleukin (IL)-33 response is dependent on Dectin-1 signaling. Effect of $H$. capsulatum (5 multiplicity of infection (MOI)) or IL-4 plus H. capsulatum on IL-33 transcription in Dectin-1 ${ }^{-1-}$ macrophages after $24 \mathrm{~h}$ of challenge. Data are normalized to uninfected wild-type (WT) macrophages. (b) Fluorescence-activated cell sorter analysis quantifying the uptake of GFP ${ }^{+}$yeasts (5 or $\left.10 \mathrm{MOI}\right)$ by WT and Dectin-1 ${ }^{-1-}$ macrophages $1 \mathrm{~h}$ after infection. (c) IL-33 mRNA expression in WT macrophages stimulated with curdlan (100 $\mu \mathrm{g} \mathrm{ml}^{-1}$ ) or IL-4 plus curdlan for $24 \mathrm{~h}$. (d) IL-33 mRNA expression in macrophages pretreated with dimethyl sulfoxide (DMSO) or Syk inhibitor (100 nM) for 90 min before exposure to H. capsulatum or IL-4 plus H. capsulatum. Values depicted in all the above experiments are mean \pm s.e.m. from 3-5 independent experiments. BM, bone marrow-derived macrophages.

protein (GFP)-expressing H. capsulatum to Dectin-1 ${ }^{-1-}$ macrophages. No differences were observed in binding of H. capsulatum yeasts to either WT or mutant macrophages at 5 or 10 MOI (Figure 5b). To substantiate the importance of Dectin-1 in IL-33 generation, macrophages were stimulated with the Dectin-1 agonist, curdlan. Treatment of WT cells with curdlan resulted in modest induction of IL-33 (4h post treatment) and a vigorous synergistic IL-33 response in the presence of IL-4 (24h posttreatment) (Figure $5 \mathrm{c}$ ).

To demonstrate the involvement of Dectin-1 signaling in the $H$. capsulatum-induced IL-33 response, we treated macrophages with the Syk-inhibitor, piceatannol and incubated them with $H$. capsulatum alone or IL-4 and H. capsulatum. A marked decrease in evocation of IL-33 was observed when cells were incubated with $H$. capsulatum or IL-4 plus $H$. capsulatum (Figure 5d). Treatment with this agent did not influence IL-4induced IL-33 transcription. The Syk inhibitor at a concentration $>20 \mu \mathrm{m}$ retards phagocytosis of $H$. capsulatum yeast particles. ${ }^{15}$ We used the inhibitor at a concentration that did not interfere with the uptake of yeasts by macrophages (data not shown). Thus these findings strongly suggest that Dectin-1 signaling is crucial for the $H$. capsulatum-induced IL-33 response in macrophages.

Blocking IL-33 signaling decreases fungal burden in vivo To determine the impact of IL-33 during $H$. capsulatum infection in CCR2 $2^{-1-}$ mice, we administered $\alpha$ ST2 antibody to one group of mice while the other received isotype control antibody. A significant lowering of fungal burden was noted in CCR2 ${ }^{-1-}$ mice that received ST2-blocking antibody at day-7 postinfection (Figure 6a). Infected WT mice do not mount an exaggerated IL-33 response and treating them with $\alpha \mathrm{ST} 2$ antibody did not lower their pulmonary fungal burden. We investigated the mechanism by which IL-33 exacerbated infection in the host. IL-33 has been shown to induce genes associated with alternative activation in macrophages. ${ }^{24} \mathrm{We}$ observed a similar upregulation in Arg-1, Chil-3, and Retnla in macrophages stimulated with IL-33 for $24 \mathrm{~h}$ (Figure 6b). Moreover, infected CCR2 ${ }^{-1-}$ mice treated with $\alpha \mathrm{ST} 2$ antibody exhibited reduced mRNA expression of alternative activation markers, in comparison with isotype control-treated mutant animals (Figure 6c). The IL-33 primed macrophages were found to be more permissive to intracellular fungal growth, in comparison with resting macrophages (Figure 6d). Thus our data indicate the detrimental attribute of IL-33 in driving an alternatively activated phenotype in phagocytes that, in turn, enhances susceptibility to H. capsulatum in CCR2 ${ }^{-1-}$ mice.

\section{DISCUSSION}

H. capsulatum-infected CCR2 ${ }^{-1-}$ mice mount an exaggerated and sustained IL-4 response that is localized to the lungs. The elevated IL-4 is associated with impaired host resistance and results in the death of mice from a progressive infection. ${ }^{3}$ In this study, we discovered a new facet of IL-4 biology using CCR2 ${ }^{-1-}$ mice infected with H. capsulatum. In a search for an 

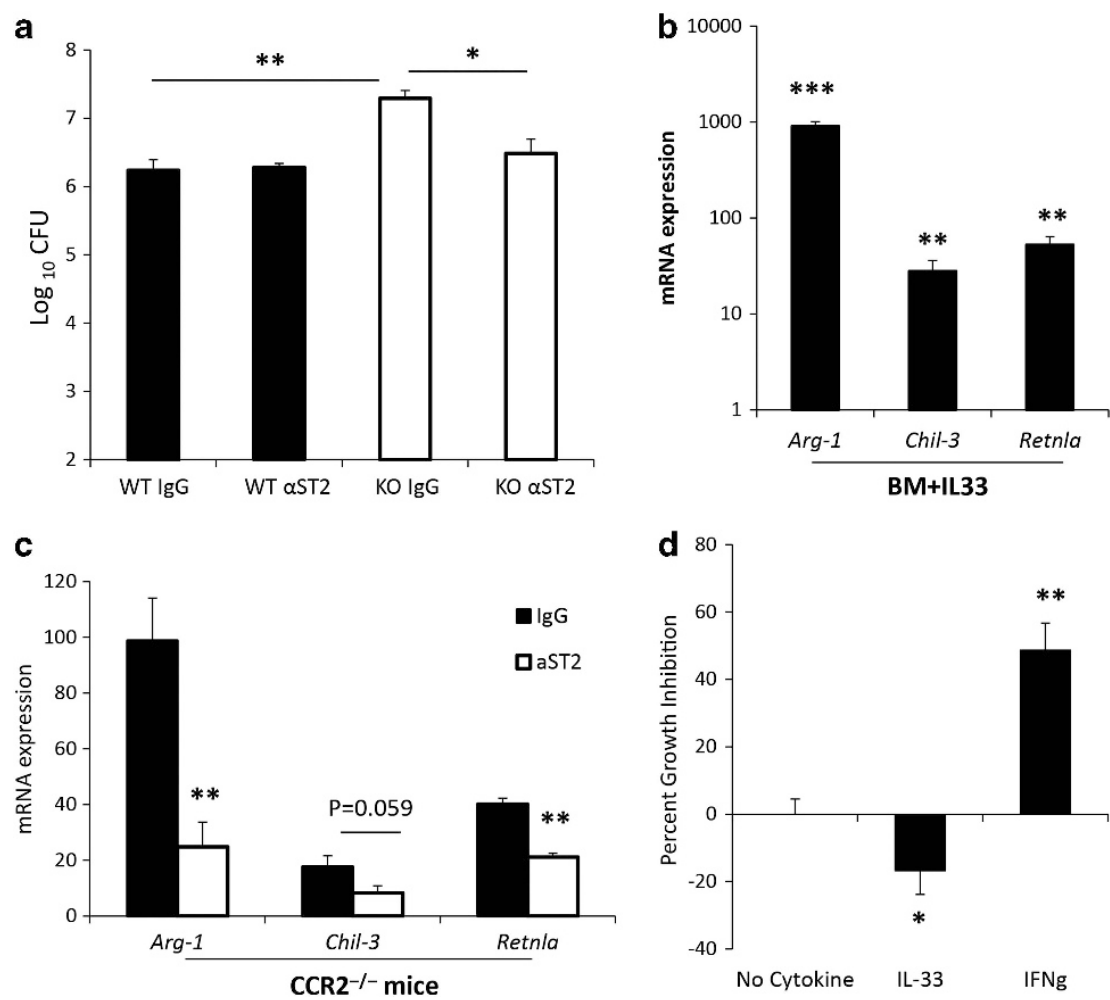

Figure 6 Detrimental effect of interleukin (IL)-33 during H. capsulatum infection. (a) Infected wild-type (WT) and CCR2 ${ }^{-1-}$ mice were intraperitoneally treated with ST2-blocking or isotype control antibody. Animals were euthanized on day 7 of infection, and pulmonary fungal burden was quantified. Values are represented as $\log _{10}$ colony-forming units (CFU) from 7-8 mice/group from two independent experiments. (b) mRNA expression of genes associated with alternatively activated phenotype in bone marrow-derived macrophages stimulated with IL-33 (10 ng ml $\left.{ }^{-1}\right)$ for $24 \mathrm{~h}$. Data are mean $\pm \mathrm{s}$.e.m. from three independent experiments; normalized to unstimulated macrophages. (c) Quantitative real-time reverse transcription PCR analysis of genes associated with alternative activation. Analysis done on mRNA from whole lung homogenates of control immunoglobulin G (IgG) or $\alpha \mathrm{ST2}$-treated CCR ${ }^{-1-}$ mice after 7 days of infection, $N=7-8$ from two independent experiments. (d) Percentage of fungal growth inhibition in unstimulated or IL-33stimulated macrophages $24 \mathrm{~h}$ after $H$. capsulatum challenge $(0.1$ multiplicity of infection). Data are normalized to fungal growth in unstimulated macrophages. Interferon (IFN)- $\gamma$-primed macrophages were used as a positive control for the growth inhibition study. Data are compiled from values of three independent experiments.

inducer of IL-4 in these mutant mice, we found that IL-4 was not a target of IL-33 but rather the converse. The latter is firmly established as a potent initiator of IL-4 synthesis in various cell populations such as basophils, natural killer T cells, and CD4 ${ }^{+}$ T cells. ${ }^{10}$ Examination of the molecular mechanism revealed that an exaggerated IL-33 response was kindled only when macrophages were exposed to IL-4 and an intracellular pathogen. The generation of IL-33 exerted a deleterious role in pulmonary mycosis as evidenced by decreased fungal burden in CCR2 ${ }^{-1-}$ mice treated with ST2-blocking antibody.

Given our previous findings in CCR $2^{-1-}$ mice and the knowledge concerning IL-33 initiating Th2 immunity, we initially sought to determine whether IL-33 instigated the exaggerated IL-4 response in CCR2 $2^{-1-}$ mice. To study the in vivo function of IL-33, we blocked interaction of this cytokine with its receptor ST2. Although antibodies may act as agonists or antagonists, this monoclonal antibody has been used to block ST2 engagment in diverse experimental models. ${ }^{19,25-27}$ In each of these studies, the antibody does not trigger an agonistic effect on the receptor ST2. Much to our surprise, generation of IL-33 in the lungs of these mice was actually observed during the onset of the adaptive phase of the immune response to
H. capsulatum rather than the innate immune phase. Even more surprising was the fact that it was macrophages that differentially produced this cytokine and not pulmonary epithelial cells. Equally peculiar, there was at least a 4-day hiatus between the time when IL- 4 was upregulated in the lungs of CCR2 $2^{-1-}$ mice (day 3 ) and the detection of enhanced IL-33 (day 7). This finding implies that the exaggerated IL-33 response by macrophages must be shaped by a cascade of interactions rather than a constitutive process. This delay was mimicked partially in vitro in which we did not observe peak IL-33 transcription until $48 \mathrm{~h}$ following exposure to IL-4.

The unexpected inverse relationship between IL- 4 and IL-33 prompted us to investigate the possibility that the former triggered an IL-33 response. Indeed, interruption of IL-4 signaling using anti-IL-4R $\alpha$ antibody diminished pulmonary IL-33 protein content in the lungs of infected CCR $2^{-1-}$ mice. Although antibody to this receptor blocks both IL-4 and IL-13, the latter is not altered in the lungs of infected CCR2 ${ }^{-1-}$ mice. ${ }^{3}$ Thus, the impact of receptor blockade is strictly on IL-4. Disrupting the same signaling pathway in WT controls did not affect IL-33 as these mice inherently mount a weaker IL-4 response in infection as compared with the mutant mice. This 
finding suggests that there exists a threshold for elicitation of IL-33 by IL- 4 . IL- 4 has been reported to evoke IL-33 in murine splenocytes and peritoneal exudate cells in vitro, but the precise mechanism was not identified. ${ }^{28}$ In concordance, we did detect a modest IL-33 response to IL-4 in uninfected macrophages in vitro that was magnified upon infection. Yet, to the best of our knowledge, IL-4-driven IL-33 in vivo has not been described.

Although epithelial cells and dendritic cells represent potential sources of IL-33 in lungs, the quantity of cytokine in these cell populations was comparable between WT and $\mathrm{CCR} 2^{-1-}$ mice. On the other hand, macrophages were the prominent producers of IL-33 in the lungs of mutant animals. An explanation for upregulation of IL-33 in the macrophage population is that alternatively activated macrophages exhibit enhanced expression and function of Dectin- $1 .{ }^{29}$ Activation of both the Dectin-1 and IL-4 signaling pathway were critical for triggering the synergistic IL-33 response. We detected intracellular IL-33 (but no extracellular release) protein that mirrored its transcription when cells were exposed to IL-4 or H. capsulatum or both. Failure to detect IL-33 in the culture supernatants was not a result of defective secretory machinery as evidenced by detecting TNF- $\alpha$ and IL- $1 \beta$ in culture supernatants but most likely a result of the lack of leader peptide sequence required for secretion of IL-33 from cells. Others have reported release of this cytokine from cells in response to external stimuli, including ATP or lysophosphotidylcholine. ${ }^{18,30}$ We did not detect the release of IL-33 from macrophages following similar treatments (data not shown). A probable consideration for the contradictory findings is that the prior studies used cell lines.

IRF-4 is a transcription factor that is engaged following activation of STAT6 and controls the expression of genes linked with alternative activation in macrophages. ${ }^{22}$ Activation of STAT6 and IRF-4 were crucial for the IL-4-prompted IL-33 response. A recent report has indicated that IRF-4 directly binds to the IL-33 locus to activate the gene. ${ }^{31} \mathrm{~A}$ similar mechanism might be in effect in macrophages stimulated with IL-4. However, an indirect role for IRF-4 in regulating IL-33 expression cannot be excluded.

Apart from its function in triggering cytokine responses to $H$. capsulatum yeast cells, Dectin-1 has been linked with recognition and innate immune responses to other fungal pathogens, such as Candida, Coccidioides, and Aspergillus. ${ }^{32-34}$ Macrophages deficient in Dectin-1 had a marked reduction in IL-33 when infected with $H$. capsulatum yeasts. This finding is congruent with a recent report implicating Dectin-1 in regulating IL-33 in an allergic model of chronic exposure to the fungus Aspergillus fumigatus. ${ }^{35}$ This study did not address the cellular source of IL-33 during the allergic phase, but its results suggest that engagement of Dectin- 1 by $\beta$-glucan on the A. fumigatus surface is critical for evoking IL-33 in the lungs. $\beta$-glucan is a vital component of $H$. capsulatum cell wall in several of the North American isolates, and engagement of this constituent by Dectin-1 activates the receptor to transduce signals that lead to IL-33 in macrophages.
H. capsulatum-prompted IL-33 is reliant on Dectin-1/Syk signaling pathway. In human neutrophils and peripheral blood mononuclear cells, engagement of IL- 4 with its receptor IL- $4 \mathrm{R} \alpha$ activates Syk kinase that ultimately augments cell adhesion and delays apoptosis. ${ }^{36}$ Thus there is a possibility that IL-4-initiated IL-33 transcription partially depends on Syk kinase. This consideration is unlikely as IL-4 stimulation of macrophages that had been previously treated with the Syk inhibitor yielded an intact IL-33 response. The synergistic elicitation of IL-33 was significantly ablated when either the IL-4 or Dectin-1 signaling pathway was disrupted. These findings strongly support that both the signaling pathways act autonomously to evoke IL-33 in a robust manner.

We demonstrated the deleterious aspect of IL-33 during intracellular fungal infection and provide proof that it contributes to host susceptibility. Others have reported adverse effects of this cytokine, but they have largely been a consequence of inducing Th 2 cytokines. In experimental cryptococcal infection or in allergic sensitization with Aspergillus or Alternaria, IL-33 is detrimental to the host by skewing the immune response to a Th2 phenotype. ${ }^{35,37,38}$ However, IL-33 did not contribute to the instigation of IL-4 response in H. capsulatum infection. Rather, the latter evoked IL-33 in the lungs to aggravate H. capsulatum infection in CCR2 ${ }^{-1-}$ mice.

One mechanism by which IL-33 predisposed these mutant mice to histoplasmosis was by triggering an alternatively activated phenotype in macrophages. Alternative activation was demonstrated previously in macrophages from infected CCR2 ${ }^{-1-}$ mice at 7 days postinfection coincident with elevated IL-33. ${ }^{3}$ Exposure of macrophages to IL-4 induces arginase-1, an enzyme that diminishes nitric oxide required for fungicidal activity. ${ }^{39}$ Moreover, IL-4 blocks the activating properties of GM-CSF and enhances intracellular zinc that supports the fungal growth. ${ }^{40,41}$ In addition to serving as an extracellular cytokine, IL-33 might function as a nuclear factor to downregulate proinflammatory genes and, in turn, augment susceptibility to intracellular infections. ${ }^{10}$

Given previous data regarding the influence of IL-4 in causing the demise of CCR2 ${ }^{-1-}$ mice, a re-examination of that work indicates that the likely cause of the collapse of immunity is attributable to IL-33. The reason why neutralization of IL-4 restored immunity was a consequence of reducing IL-33, rather than exerting an IL-33-independent effect. This finding represents a fresh paradigm in Th2 immunity as IL-33 is principally an initiator of IL- 4 and other Th2 cytokines. The work establishes the existence of a novel positive feedback loop between two key type II immunity-inducing cytokines. The knowledge from this study can be extrapolated to other intracellular infections in which IL-4 dampens protective immunity. Indeed, an IL-4/IL-33 axis could exist that exacerbates infection, and IL-33 may be target for interdiction in progressive intracellular infections. Our findings are important for non-infectious Th2 diseases, including allergic asthma and eczema, where a similar IL-4/IL-33 coupling might be in effect. Our work highlights the detrimental effect of IL-33 
in host defenses to an intracellular pathogen. Comprehensive understanding of how exaggerated amounts of IL-33 condition the host to be more susceptible to $H$. capsulatum or other intracellular pathogens is a necessary pursuit.

\section{METHODS}

Mice. Male C57BL/6 and breeding pairs of CCR2 ${ }^{-1-}$ (C57BL/6 background) mice were purchased from The Jackson Laboratory, Bar Harbor, ME. Bone marrow cells from IL- $4^{-1-}$, IL- $4 \mathrm{R} \alpha^{-1-}$, and STAT $^{-1-}$ mice (C57BL/6 background) were generously provided by Senad Divanovic (Cincinnati Childrens' Hospital Medical Center, Cincinnati, OH). Bone marrow cells from Dectin-1 $1^{-1-}$ and IRF-4 ${ }^{-1-}$ mice were a gift from Stuart Levitz at University of Massachusetts, Worcester, MA and Lu Runqing at University of Nebraska, Omaha, NE, respectively. Animals were housed in isolator cages and maintained by the Department of Laboratory Animal Medicine, accredited by the Association for Assessment and Accreditation of Laboratory Animal Care. All animal experiments were performed in accordance with the Animal Welfare Act guidelines of the National Institutes of Health, and all protocols were approved by the Institutional Animal Care and Use Committee of the University of Cincinnati.

Preparation of $\boldsymbol{H}$. capsulatum and infection of mice. $H$. capsulatum yeast strain $\mathrm{G} 217 \mathrm{~B}$ and $\mathrm{GFP}^{+}$yeasts were grown for $72 \mathrm{~h}$ at $37^{\circ} \mathrm{C}$ as described. ${ }^{42}$ To infect mice, 6-8-week-old animals were inoculated intranasally with $2 \times 10^{6}$ yeasts in $\sim 30 \mu \mathrm{l}$ of Hank's Balanced Salt Solution (HBSS; HyClone, Logan, UT).

Organ culture for $\boldsymbol{H}$. capsulatum. Organs were homogenized in sterile HBSS and serially diluted and plated onto mycosel-agar plates containing 5\% sheep blood and 5\% glucose. Plates were incubated at $30^{\circ} \mathrm{C}$. The limit of detection was $10^{2}$ CFU.

In vivo blocking of IL-4R $\alpha$ and T1/ST2. For blocking IL- $4 \mathrm{R} \alpha$, mice were injected intraperitoneally with $500 \mu \mathrm{g}$ of rat anti-mouse IL-4R $\alpha$ (AMGEN, Thousand Oaks, CA) or control antibody (Bio X Cell, Lebanon, $\mathrm{NH}$ ) on day 0. For disrupting IL-33 signaling, mice were injected with $300 \mu \mathrm{g}$ of rat anti-mouse T1/ST2 antibody (AMGEN) on days 0 and 3 of infection.

Isolation of lung leukocytes. Lungs were homogenized with the gentleMACS dissociator (Miltenyi Biotec, Auburn, CA) in $5 \mathrm{ml}$ of HBSS with $2 \mathrm{mg} \mathrm{ml}^{-1}$ of collagenase D (Roche, Mannheim, Germany) and $40 \mathrm{U} \mathrm{ml}^{-1}$ of DNase I (Roche) for $30 \mathrm{~min}$ at $37^{\circ} \mathrm{C}$. The homogenate was percolated through a $60-\mu \mathrm{m}$ nylon mesh (Spectrum Laboratories, Rancho Dominguez, CA) and washed three times with HBSS. Leukocytes were isolated by separation on Lympholyte $M$ (Cedarlane Laboratories, Burlington, ON).

Flow cytometry and cell sorting. The phenotype of cells from mouse lungs was determined by incubating lung leukocytes with the indicated antibodies and CD16/32 to limit nonspecific binding. Leukocytes were stained at $4{ }^{\circ} \mathrm{C}$ for $15 \mathrm{~min}$ in phosphate-buffered saline containing $1 \%$ bovine serum albumin and $0.01 \%$ sodium azide. Cells were stained with combinations of the following antibodies: FITC-conjugated I-A ${ }^{\mathrm{b}}$; PE-conjugated CD11c; PerCP-conjugated CD45; and APC-conjugated F4/80 and EpCAM from BD Biosciences (San Jose, CA). For intracellular IL-33 staining, cells were incubated with Cytofix/ Cytoperm (BD Biosciences), washed in Permeabilization Buffer (BD Biosciences), and stained for 30 min with PE-conjugated IL-33 (R\&D systems, Minneapolis, MN). Cells were washed and resuspended in 1\% paraformaldehyde. Isotype controls were used. Data were acquired using BD Accuri C6 cytometer (San Jose, CA) and analyzed using the FCS Express 4.0 Software (DeNovo Software, Los Angeles, CA). For cell sorting experiments, $\mathrm{F} 4 / 80^{+}$leukocytes from the lungs of WT and CCR2 ${ }^{-1-}$ mice were isolated at day-7 postinfection using 5 -laser FACS Aria II (BD Biosciences).
Generation of bone marrow-derived, peritoneal, and alveolar macrophages. Bone marrow was isolated from tibiae and femurs of 6-10-week-old mice by flushing with HBSS. Cells were dispensed into tissue culture flasks at a density of $2 \times 10^{5}$ cells ml $^{-1}$ of RPMI-1640 supplemented with $10 \%$ fetal bovine serum, $0.1 \%$ gentamicin sulfate, $5 \times 10^{-5} \mathrm{M} 2$-mercaptoethanol, and $10 \mathrm{ng} \mathrm{ml}^{-1}$ of mouse GM-CSF (Peprotech, Rocky Hill, NJ). Flasks were incubated at $37^{\circ} \mathrm{C}$ in $5 \% \quad \mathrm{CO}_{2}$. Macrophages were harvested at day 7. Non-adherent cells were removed, and trypsin-EDTA was added for $10 \mathrm{~min}$ at $37^{\circ} \mathrm{C}$. Cells were collected, washed with HBSS, and dispensed into culture dishes. Resident peritoneal macrophages from mice were isolated and adhered overnight. The following day, non-adherent cells were removed. Alveolar macrophages were isolated by lavaging the lungs of uninfected WT mice with phosphate-buffered saline. Non-adherent cells were washed off, and adherent macrophages were used.

Binding of $\boldsymbol{H}$. capsulatum yeast to macrophages. Bone marrowderived macrophages were seeded in 12 -well plates and cultured overnight. The following day, plates were cooled on ice for $20 \mathrm{~min}$ before the addition of non-opsonized GFP ${ }^{+} H$. capsulatum to the wells at final yeast: macrophage ratio of $5: 1$ or 10:1. The plates were placed on a shaker at a rotation speed of 150 r.p.m. for 2 min and left on ice for another $60 \mathrm{~min}$. This process allowed GFP-labeled yeasts to settle on and contact with macrophages before phagocytosis took place. The plates were then placed in a $\mathrm{CO}_{2}$ incubator to allow phagocytosis to have a synchronous start. After 60 min of incubation at $37^{\circ} \mathrm{C}$, cells were washed twice in warm HBSS. Macrophages were then detached by treatment with cell dissociation buffer (Invitrogen, Carlsbad, CA) and fixed in $1 \%$ paraformaldehyde. Finally, to determine the Association Index, the percentages of $\mathrm{GFP}^{+}$cells were quantified by flow cytometry.

In vitro culture conditions. For most experiments, bone marrowderived, peritoneal, or alveolar macrophages (except the control or $H$. capsulatum only group) were primed with $10 \mathrm{ng} \mathrm{ml}^{-1}$ of IL-4 (Peprotech) for $24 \mathrm{~h}$. Subsequently, fresh media was replenished, and macrophages were subjected to IL-4 or H. capsulatum or both for $24 \mathrm{~h}$. For inhibition studies, Piceatannol (TOCRIS, Bristol, UK) was added to macrophages $90 \mathrm{~min}$ before infection.

RNA Isolation, cDNA synthesis, and quantitative real-time reverse transcription PCR. Total RNA from whole lungs of mice was isolated using TRIzol (Invitrogen) and from in vitro macrophage cultures using the RNeasy Kit (Qiagen, Valencia, CA). Oligo(dT)-primed cDNA was prepared by using the reverse transcriptase system (Promega, Madison, WI). Quantitative real-time reverse transcription PCR analysis was performed using TaqMan master mixture and primers obtained from Applied Biosystems (Foster City, CA). Samples were analyzed with ABI Prism 7500. The hypoxanthine phosphoribosyl transferase housekeeping gene was used as an internal control. The conditions for amplification were $50{ }^{\circ} \mathrm{C}$ for $2 \mathrm{~min}$ and $95^{\circ} \mathrm{C}$ for $10 \mathrm{~min}$, followed by 40 cycles of $95^{\circ} \mathrm{C}$ for $15 \mathrm{~s}$ and $60^{\circ} \mathrm{C}$ for $1 \mathrm{~min}$.

Growth-inhibition assays. One hundred thousand bone marrowderived macrophages were plated on a 96-well in culture media with or without IL-33 $\left(10 \mathrm{ng} \mathrm{ml}^{-1}\right)$ for $24 \mathrm{~h}$. The following day, cells were infected with $0.1 \mathrm{MOI}$ of yeasts. Fungal growth was assayed $24 \mathrm{~h}$ after infection. Macrophages were lysed, and yeasts were plated. Colonies were counted 10 days later.

Measurement of IL-33 by enzyme-linked immunosorbent assay. IL-33 protein was quantified in lung homogenates and macrophage cell lysates (lysed using deionized water) by using an enzyme-linked immunosorbent assay kit (R\&D Systems).

Statistics. Analysis of variance with Dunn's test was used to compare multiple groups, while Student's $t$-test was used to compare two 
groups. $P$-value of $<0.05$ was considered significant. For all graphs, ${ }^{\star} P=0.01-0.05,{ }^{* *} P=0.005-0.01$, and ${ }^{* * *} P<0.005$.

SUPPLEMENTARY MATERIAL is linked to the online version of the paper at http://www.nature.com/mi

\section{ACKNOWLEDGMENTS}

We would like to thank Dr K.S. Vignesh and W. Buesing for technical help with the experiments. We thank $\mathrm{Dr} \mathrm{H}$. Singh for his valuable suggestions and J. Mohammed for sorting and flow cytometry support. This work was supported by an $\mathrm{NIH}$ grant $\mathrm{Al}-083313$ from the National Institute of Allergy and Infectious Diseases.

\section{DISCLOSURE}

The authors declared no conflict of interest.

(c) 2015 Society for Mucosal Immunology

\section{REFERENCES}

1. Finkelman, F.D. \& Urban, J.F. Jr. The other side of the coin: the protective role of the TH2 cytokines. J. Allergy Clin. Immunol. 107, 772-780 (2001).

2. Gause, W.C., Wynn, T.A. \& Allen, J.E. Type 2 immunity and wound healing: evolutionary refinement of adaptive immunity by helminths. Nat. Rev. Immunol. 13, 607-614 (2013).

3. Szymczak, W.A. \& Deepe, G.S. Jr. The CCL7-CCL2-CCR2 axis regulates IL-4 production in lungs and fungal immunity. J. Immunol. 183, 1964-1974 (2009).

4. Kahnert, A. et al. Alternative activation deprives macrophages of a coordinated defense program to Mycobacterium tuberculosis. Eur. J. Immunol. 36, 631-647 (2006).

5. Perkins, C., Wills-Karp, M. \& Finkelman, F.D. IL-4 induces IL-13independent allergic airway inflammation. J. Allergy Clin. Immunol. 118, 410-419 (2006).

6. He, R. et al. TSLP acts on infiltrating effector T cells to drive allergic skin inflammation. Proc. Natl. Acad. Sci. USA 105, 11875-11880 (2008).

7. Shirey, K.A., Cole, L.E., Keegan, A.D. \& Vogel, S.N. Francisella tularensis live vaccine strain induces macrophage alternative activation as a survival mechanism. J. Immunol. 181, 4159-4167 (2008).

8. Traynor, T.R., Kuziel, W.A., Toews, G.B. \& Huffnagle, G.B. CCR2 expression determines T1 versus T2 polarization during pulmonary Cryptococcus neoformans infection. J. Immunol. 164, 2021-2027 (2000).

9. Pulendran, B. \& Artis, D. New paradigms in type 2 immunity. Science 337 , 431-435 (2012).

10. Sattler, S., Smits, H.H., Xu, D. \& Huang, F.P. The evolutionary role of the IL-33/ST2 system in host immune defence. Arch. Immunol. Ther. Exp. Warsz) 61, 107-117 (2013)

11. Allendoerfer, R. \& Deepe, G.S. Jr. Infection with Histoplasma capsulatum: host-fungus interface. Rev. Iberoam Micol. 15, 256-260 (1998).

12. Brown, G.D. et al. Hidden killers: human fungal infections. Sci. Transl. Med. 4, 165rv113 (2012).

13. Wuthrich, M., Deepe, G.S. Jr. \& Klein, B. Adaptive immunity to fungi. Annu. Rev. Immunol. 30, 115-148 (2012).

14. Newman, S.L., Bucher, C., Rhodes, J. \& Bullock, W.E. Phagocytosis of Histoplasma capsulatum yeasts and microconidia by human cultured macrophages and alveolar macrophages. Cellular cytoskeleton requirement for attachment and ingestion. J. Clin. Invest. 85, 223-230 (1990).

15. Lin, J.S., Huang, J.H., Hung, L.Y., Wu, S.Y. \& Wu-Hsieh, B.A. Distinct roles of complement receptor 3 , Dectin-1, and sialic acids in murine macrophage interaction with Histoplasma yeast. J. Leukoc. Biol. 88, 95-106 (2010).

16. Kroetz, D.N. \& Deepe, G.S. The role of cytokines and chemokines in Histoplasma capsulatum infection. Cytokine 58, 112-117 (2012).

17. Paul, W.E. \& Zhu, J. How are $T(H) 2$-type immune responses initiated and amplified?. Nat. Rev. Immunol. 10, 225-235 (2010).

18. Kouzaki, H., lijima, K., Kobayashi, T., O'Grady, S.M. \& Kita, H. The danger signal, extracellular ATP, is a sensor for an airborne allergen and triggers IL-33 release and innate Th2-type responses. J. Immunol. 186, 4375-4387 (2011)
19. Wills-Karp, M. et al. Trefoil factor 2 rapidly induces interleukin 33 to promote type 2 immunity during allergic asthma and hookworm infection. J. Exp. Med. 209, 607-622 (2012).

20. Chang, Y.J. et al. Innate lymphoid cells mediate influenza-induced airway hyper-reactivity independently of adaptive immunity. Nat. Immunol. 12, 631-638 (2011).

21. Walford, H.H. \& Doherty, T.A. STAT6 and lung inflammation. JAKSTAT 2, e25301 (2013).

22. El Chartouni, C., Schwarzfischer, L. \& Rehli, M. Interleukin-4 induced interferon regulatory factor (Irf) 4 participates in the regulation of alternative macrophage priming. Immunobiology 215, 821-825 (2010).

23. Qualls, J.E. et al. Arginine usage in mycobacteria-infected macrophages depends on autocrine-paracrine cytokine signaling. Sci Signal 3, ra62 (2010).

24. Yang, Z. et al. Macrophages as IL-25/L-33-responsive cells play an important role in the induction of type 2 immunity. PLoS One 8, e59441 (2013).

25. Palmer, G. et al. Inhibition of interleukin-33 signaling attenuates the severity of experimental arthritis. Arthritis Rheum. 60, 738-749 (2009).

26. Sedhom, M.A. et al. Neutralisation of the interleukin-33/ST2 pathway ameliorates experimental colitis through enhancement of mucosal healing in mice. Gut 62, 1714-1723 (2013).

27. Li, J. et al. Biliary repair and carcinogenesis are mediated by IL-33dependent cholangiocyte proliferation. J. Clin. Invest. 124, 3241-3251 (2014).

28. Zhao, W.H. \& Hu, Z.Q. Up-regulation of IL-33 expression in various types of murine cells by IL-3 and IL-4. Cytokine 58, 267-273 (2012).

29. Willment, J.A. et al. Dectin-1 expression and function are enhanced on alternatively activated and GM-CSF-treated macrophages and are negatively regulated by IL-10, dexamethasone, and lipopolysaccharide. J. Immunol. 171, 4569-4573 (2003).

30. Palm, N.W. et al. Bee venom phospholipase A2 induces a primary type 2 response that is dependent on the receptor ST2 and confers protective immunity. Immunity 39, 976-985 (2013).

31. Williams, J.W. et al. Transcription factor IRF4 drives dendritic cells to promote Th2 differentiation. Nat. Commun. 4, 2990 (2013).

32. LeibundGut-Landmann, S. et al. Syk- and CARD9-dependent coupling of innate immunity to the induction of T helper cells that produce interleukin 17. Nat. Immunol. 8, 630-638 (2007).

33. Steele, C. et al. The beta-glucan receptor dectin-1 recognizes specific morphologies of Aspergillus fumigatus. PLoS Pathog. 1, e42 (2005).

34. Viriyakosol, S., Jimenez Mdel, P., Gurney, M.A., Ashbaugh, M.E. \& Fierer, J. Dectin-1 is required for resistance to coccidioidomycosis in mice. MBio 4, e00597-00512 (2013).

35. Lilly, L.M. et al. The beta-glucan receptor dectin-1 promotes lung immunopathology during fungal allergy via IL-22. J. Immunol. 189, 3653-3660 (2012).

36. Ennaciri, J. \& Girard, D. IL-4R(alpha), a new member that associates with Syk kinase: implication in IL-4-induced human neutrophil functions. J. Immunol. 183, 5261-5269 (2009).

37. Flaczyk, A. et al. IL-33 signaling regulates innate and adaptive immunity to Cryptococcus neoformans. J. Immunol. 191, 2503-2513 (2013).

38. Doherty, T.A. et al. STAT6 regulates natural helper cell proliferation during lung inflammation initiated by Alternaria. Am. J. Physiol. Lung Cell. Mol. Physiol. 303, L577-L588 (2012).

39. Davis, M.J. et al. Macrophage M1/M2 polarization dynamically adapts to changes in cytokine microenvironments in Cryptococcus neoformans infection. MBio 4, e00264-00213 (2013).

40. Subramanian Vignesh, K., Landero Figueroa, J.A., Porollo, A., Caruso, J.A. \& Deepe, G.S. Jr. Granulocyte macrophage-colony stimulating factor induced Zn sequestration enhances macrophage superoxide and limits intracellular pathogen survival. Immunity 39, 697-710 (2013).

41. Winters, M.S., Chan, Q., Caruso, J.A. \& Deepe, G.S. Jr. Metallomic analysis of macrophages infected with Histoplasma capsulatum reveals a fundamental role for zinc in host defenses. J. Infect. Dis. 202, 1136-1145 (2010).

42. Allendoerfer, R. \& Deepe, G.S. Jr. Intrapulmonary response to Histoplasma capsulatum in gamma interferon knockout mice. Infect. Immun. 65, 2564-2569 (1997) 